\title{
Macroscopic Quantum State Analyzed Particle by Particle
}

\author{
Federica A. Beduini, ${ }^{1}$ Joanna A. Zielińska, ${ }^{1}$ Vito G. Lucivero, ${ }^{1}$ Yannick A. de Icaza Astiz, ${ }^{1}$ and Morgan W. Mitchell ${ }^{1,2, *}$ \\ ${ }^{1}$ ICFO-Institut de Ciencies Fotoniques, Mediterranean Technology Park, 08860 Castelldefels (Barcelona), Spain \\ ${ }^{2}$ ICREA-Institució Catalana de Recerca i Estudis Avançats, 08015 Barcelona, Spain
}

(Received 15 January 2015; published 27 March 2015)

\begin{abstract}
Macroscopic quantum phenomena, e.g., superconductivity and squeezing, are believed to result from entanglement of macroscopic numbers of particles. We report the first direct study of this kind of entanglement: we use discrete quantum tomography to reconstruct the joint quantum state of photon pairs extracted from polarization-squeezed light. Our observations confirm several predictions from spinsqueezing theory [Beduini et al., Phys. Rev. Lett. 111, 143601 (2013)], including strong entanglement and entanglement of all photon pairs within the squeezing coherence time. This photon-by-photon analysis may give insight into other macroscopic many-body systems, e.g., photon Bose-Einstein condensates.
\end{abstract}

DOI: 10.1103/PhysRevLett.114.120402

Measurement of the individual components of a macroscopic quantum system is a central goal of quantum simulation [1] and promises to reveal how exotic macroscopic phenomena emerge from large-scale entanglement [2-5]. In both natural and synthetic quantum systems, however, detection of individual quantum states remains extremely challenging [6-8]. Squeezing phenomena, in which fluctuations in a macroscopic variable are reduced below the naive quantum limits, offer a privileged window into the role of entanglement in macroscopic quantum phenomena. Spin-squeezing inequalities (SSIs) demarcate the boundary between squeezed states and classical states, i.e., between macroscopic behavior producible with and without entanglement. Beyond detecting entanglement [9], SSIs can quantify entanglement depth [5] and the number of entangled particles [10]. Spin-squeezing experiments $[11,12]$ have used SSIs to claim 500000 entangled atoms and entanglement depth of 170 . These claims far exceed the records with individually controlled particles: 14 trapped ions [13] and 8 photons [14].

Here we report the first study of entangled particles underlying a macroscopic quantum phenomenon and the first test of a SSI as a predictor of microscopic entanglement. While SSIs have mostly been applied to atomic spin squeezing [11,12], a direct test with atoms appears challenging. In contrast to individually trapped atoms or ions, detection of atoms from ensembles $[7,8,15,16]$ has not yet shown the simultaneous, individual, state-selective detection required for multiparticle state characterization [17]. Here we exploit the strengths of photonic technology, including high coherence and efficient single-particle detection, to study the predicted large-scale entanglement [18] underlying the macroscopic quantum phenomenon of polarization squeezing [19]. We generate a polarizationsqueezed beam, extract photon pairs, and tomographically reconstruct [20] their joint quantum state as a function of their temporal separation. The results confirm several
PACS numbers: 03.65.Ta, 03.65.Ud, 03.67.Bg, 42.50.Dv

predictions of spin-squeezing theory: all photons arriving within the squeezing coherence time are entangled, and, counterintuitively, increased squeezing can reduce bipartite entanglement. The results provide direct evidence for entanglement of macroscopic numbers of particles [18] and introduce microanalysis to the study of macroscopic quantum phenomena.

Photons are becoming an attractive system for studying strongly correlated bosons, including Bose-Einstein condensation (BEC) [21], nonlinear Josephson oscillations [22], dynamical squeezing [23], and strong Rydbergatom-mediated interactions [24]. Recent work has probed photon-BEC thermodynamics through photon-number statistics [25], and there are proposals to use photons for lattice-gas quantum simulators [26]. Here we add the capability for microstate analysis to the photonic quantum gas toolkit. We use the photon polarization as a binary degree of freedom analogous to spin- $1 / 2$ atoms.

Nonclassical polarization correlations and photon entanglement are related through the second-order correlation functions $\quad R_{i j, m n}^{(2)}(\tau) \equiv\left\langle\hat{a}_{i}^{\dagger}(t) \hat{a}_{j}^{\dagger}(t+\tau) \hat{a}_{n}(t+\tau) \hat{a}_{m}(t)\right\rangle$, where the $\hat{a}_{i}^{\dagger}(t)$ are mode operators and subscripts indicate $H$ or $V$ polarization [18]. Classically, these obey the Cauchy-Schwarz-like inequalities

$$
\begin{aligned}
\left|R_{H H, V V}^{(2)}(\tau)\right|^{2} & \leq R_{H V, H V}^{(2)}(\tau) R_{V H, V H}^{(2)}(\tau), \\
\left|R_{H V, V H}^{(2)}(\tau)\right|^{2} & \leq R_{H H, H H}^{(2)}(\tau) R_{V V, V V}^{(2)}(\tau),
\end{aligned}
$$

which can be violated by squeezed fields. At the same time, these correlation functions give the two-photon density matrix $\rho^{(2)}$ via Glauber photodetection theory, $\rho_{i j, k l}^{(2)}(\tau) \propto$ $R_{i j, k l}^{(2)}(\tau)$ [18]. For a polarization-squeezed (PS) state consisting of vertically polarized $(V)$ squeezed vacuum and a stationary horizontally polarized $(H)$ coherent field, the density matrix is a so-called $X$ state in the 
$\{H H, H V, V H, V V\}$ basis, i.e., with non-null elements only along the two diagonals,

$$
\rho^{(2)} \propto\left(\begin{array}{cccc}
R_{H H, H H}^{(2)} & 0 & 0 & R_{H H, V V}^{(2)} \\
0 & R_{H V, H V}^{(2)} & R_{H V, V H}^{(2)} & 0 \\
0 & R_{V H, H V}^{(2)} & R_{V H, V H}^{(2)} & 0 \\
R_{V V, H H}^{(2)} & 0 & 0 & R_{V V, V V}^{(2)}
\end{array}\right),
$$

where we have suppressed the $\tau$ dependence for clarity. $\rho^{(2)}$ is nonpositive under partial transpose and, thus, entangled by the Peres-Horodecki criterion if either of Eqs. (1a) or (1b) is violated, i.e., for polarization-squeezed states. In other words, this type of polarization squeezing requires an underlying photonic entanglement.

Density matrices $\rho^{(2)}(\tau)$ are given explicitly in Ref. [18] as a function of $\Phi_{C}$ and $\Phi_{S}$, the photon fluxes in the coherent and squeezed components of the PS state, respectively. The predicted $\rho^{(2)}(\tau)$ shows large concurrence, up to $100 \%$, for pure squeezed vacuum (SV) with low squeezing, i.e., $\Phi_{S} \ll \Gamma$, where $\Gamma$ is the bandwidth of the squeezed vacuum [18]. In these conditions, the concurrence is large for a region defined by

$$
\Gamma \Phi_{S} \approx \Phi_{C}^{2}, \quad \Phi_{S}<\Gamma, \quad \tau \Gamma<1 .
$$

This geometry reflects the fact that the entanglement arises from two-photon interference, which is strongest when the two-photon contributions from the $H$ and $V$ states are similar, i.e., when $\rho_{H H, H H}^{(2)}(\tau) \approx \rho_{V V, V V}^{(2)}(\tau)$.

The apparatus to produce PS states and detect photon pairs extracted from them is shown in Fig. 1(a). The state is generated using a polarizing beam splitter to combine an $H$-polarized coherent state into the same spatial mode as a $V$-polarized squeezed vacuum state with the same frequency. The SV is the output of the degenerate mode of a type-I optical parametric oscillator (OPO) [27]. The relative phase of the $H$ and $V$ inputs is stabilized with the help of a "seed beam" injected into the OPO, as described in the Supplemental Material [28]. A stable phase $\varphi$ between the $H$ and $V$ component is necessary to obtain entangled states: if the corner off-diagonal elements, namely, $\rho_{H H, V V}$ and its Hermitian conjugate $\rho_{V V, H H}$ vanish, also the concurrence goes to zero. As $\rho_{H H, V V} \propto e^{i \varphi}$, when the phase is fluctuating freely during the photon acquisition, the tomography reconstruction procedure yields the average value of $\rho_{H H, V V}$, which is equal to zero in case of fast phase drifts compared to the acquisition time (4-5 h long). During the measurement, we stabilize the length of both the coherent and the OPO pump path with active feedback on the position of one mirror in each path: this maintains $\varphi$ stable within a few degrees for hours.

We reconstruct the polarization state of the photon pairs belonging to the PS state by discrete quantum tomography
[17]. Our polarization analyzer consists of a quarter-wave plate (QWP) and a half-wave plate (HWP) at angles $\theta_{\mathrm{QWP}}, \theta_{\mathrm{HWP}}$, respectively, followed by coupling into a polarization-maintaining fiber with fast axis aligned to the $H$ polarization. The fast and slow polarizations are then separated with a calcite beam displacer, filtered (see below), split with 50:50 fiber beam splitters (FBSs), and detected with single-photon avalanche photodiodes (SPADs). A multichannel time-stamping board and postprocessing are used to record arrival times. Seven sets of QWP and HWP angles listed in the Supplemental Material [28] are used to detect in seven distinct polarization bases, and for each basis, we can collect both same-polarization and mixed-polarization coincidences. We recover the photon pair density matrix with a maximum likelihood algorithm [20] for each delay $\tau$.

We use a Faraday anomalous dispersion atomic filter (FADOF) [29] to eliminate photons in nondegenerate OPO modes, which would otherwise be detected by the broadband SPADs. The filter has a transmission bandwidth of $223 \mathrm{MHz}$ HWHM, narrower than the OPO's $500 \mathrm{MHz}$ free spectral range, rejects the $\sim 600$ out-of-band modes at the $1: 10^{5}$ level, and has been measured to give an output consisting of at least $96 \%$ degenerate-mode photons [30].

Both phase stabilization and quantum tomography involve the measurement of the PS state, but they cannot happen simultaneously, as any attempt at splitting the state would reduce both the degree of squeezing and its purity. We use a galvanometer mirror to switch rapidly $(\sim 100 \mathrm{~Hz})$ the PS state between the tomography and the phase stabilization setup to acquire photons in $\sim 3 \mathrm{~ms}$ intervals, small compared to the few-second time scale of phase drifts in the system.

Density matrices for different values of $\tau$ are shown in Fig. 1(b), for $\Phi_{C}=9.6 \times 10^{5} \mathrm{ph} / \mathrm{s}$ and $\Phi_{S}=$ $1.9 \times 10^{5} \mathrm{ph} / \mathrm{s}$. These show the predicted " $X$ " shape, apart from small but nonzero coherences off of the two diagonals, e.g., $\rho_{V V, V H}$. These are most probably generated by experimental imperfections, such as leakage of CS light into the SV polarization, e.g., by defects in the combining polarizing beam splitter (PBS) or FBS. The density matrices show strong $\rho_{H H, V V}$ coherences, giving a good fidelity with a NooN-like state of the form $\cos \theta|H H\rangle+\sin \theta|V V\rangle$ [31]. Figure 1(c) shows the relationship between concurrence (top graph) and the relative strength of the $|H H\rangle$ and $|V V\rangle$ components (bottom graph). This confirms that maximum concurrence occurs when the two-photon interference between $H$ and $V$ polarization is maximum, i.e., $\rho_{H H, H H}^{(2)}(\tau) \approx \rho_{V V, V V}^{(2)}(\tau)$ [see Eq. (3)]. Because $\rho_{V V, V V}^{(2)}(\tau)$ drops off exponentially with $\tau$ while $\rho_{H H, H H}^{(2)}(\tau)$ is $\tau$ independent, the concurrence necessarily drops off for photons widely separated in time. The concurrence values shown give lower bounds on the entanglement of the photons in the squeezed state: decoherence due to experimental limitations, e.g., noise 
(a)

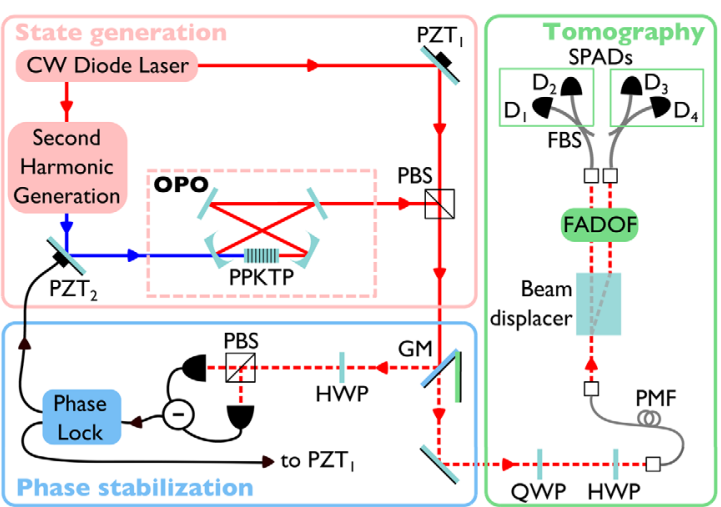

(d)

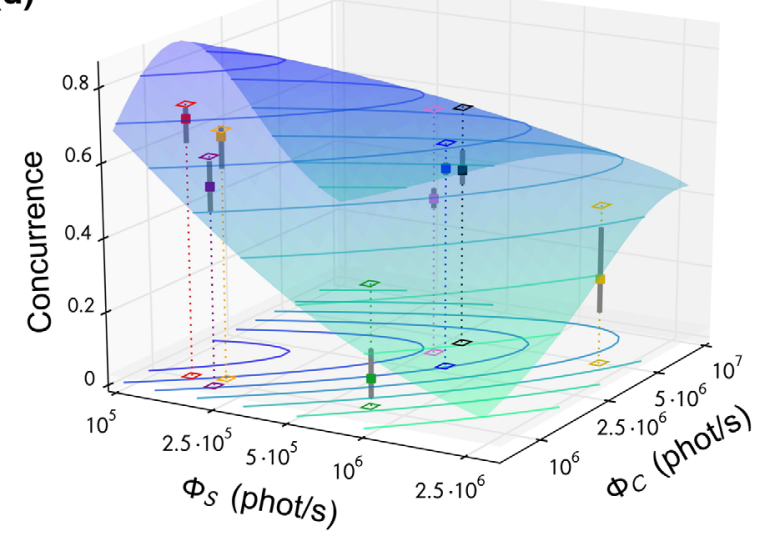

(b)
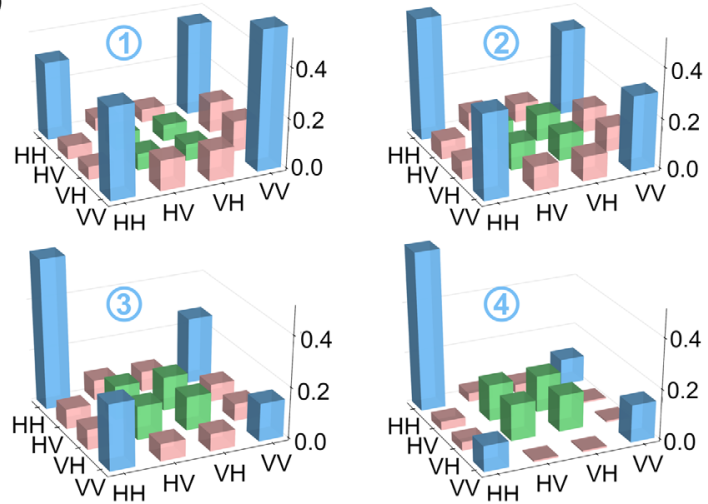

(c)

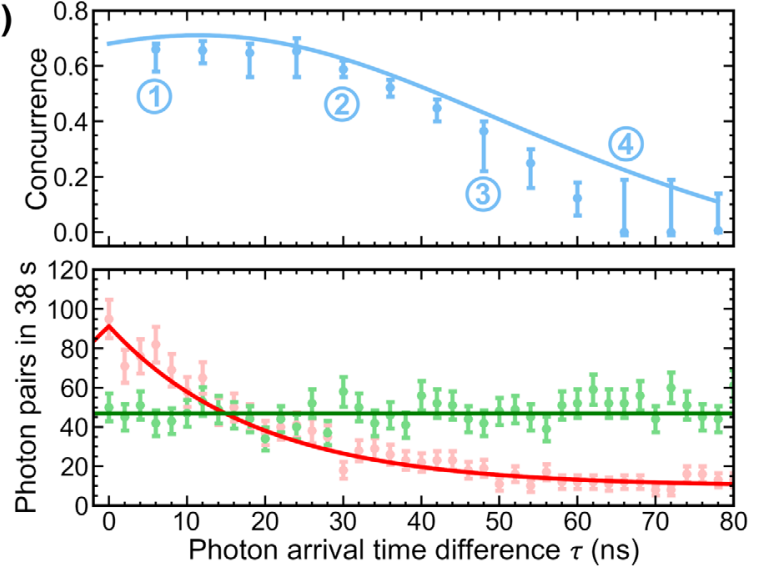

FIG. 1 (color online). Photon-level analysis of a polarization-squeezed state. (a) Simplified experimental schematic (full schematic in the Supplemental Material [28]). (red box) A $795 \mathrm{~nm}$ continuous-wave laser is locked to the $\mathrm{Rb} D_{1}$ line, frequency doubled, and used to pump a subthreshold optical parametric oscillator (OPO) producing $795 \mathrm{~nm}$ squeezed vacuum (SV) with $V$ polarization. A portion of the laser is also taken to produce an $H$ polarization coherent state (CS), and the two components are combined on a polarizing beam splitter (PBS) to produce PS light. Two piezoelectric actuators (PZTs) act on the relative phase of the SV and CS components of the state. (blue box) A galvanometer mirror (GM) directs the PS beam alternately to a continuous-variable (CV) detector, sensitive to the macroscopic polarization of the state or to a discrete-variable (DV) photon-counting system. The CV detector consists of a half wave plate (HWP), PBS, and linear differential detector. Its electronic signals are fed back to $\mathrm{PZT}_{1}$ and $\mathrm{PZT}_{2}$ to stabilize the $\mathrm{SV}$ and $\mathrm{CS}$ relative phase. (green box) The DV detector consists of a quarter wave plate (QWP), HWP, polarization-maintaining fiber (PMF), and beam displacer, together acting as a polarization analyzer splitting the two polarizations (in a basis determined by the QWP and HWP) to two parallel output channels. A Faraday anomalous-dispersion optical filter (FADOF) is used to eliminate photons from nondegenerate modes of the OPO. The two output paths are each split to two single-photon avalanche photodiodes (SPADs) to allow detection of both same- and opposite-polarization photon pairs. (b) Reconstructed density matrices (magnitudes only) of photons extracted from a PS state with $\Phi_{C}=9.6 \times 10^{5} \mathrm{ph} / \mathrm{s}$ and $\Phi_{S}=1.9 \times 10^{5} \mathrm{ph} / \mathrm{s}$ [orange square in (d)] for mean arrival time differences $\langle|\tau|\rangle=6,30,48$, and $66 \mathrm{~ns}$. We include all events within the coincidence window $\langle|\tau|\rangle-6 \mathrm{~ns} \leq|\tau| \leq\langle|\tau|\rangle+6 \mathrm{~ns}$. Blue bars indicate the "NooN" portion of the state, a superposition of $|H H\rangle$ and $|V V\rangle$, green bars indicate the " $W$ " portion of the state, $\propto|H V\rangle+|V H\rangle$, and pink bars indicate anomalous coherences (see text). (c) (upper) Concurrence of the two-photon density matrices versus $\langle|\tau|\rangle$ under the same conditions. Error bars indicate $\pm 1 \sigma$ statistical error estimated by a bootstrapping procedure (see the Supplemental Material). Results show entanglement for photons separated by up to $60 \mathrm{~ns}$, as predicted by the theory (solid line). (lower) SV (red) and CS (green-flat line) contributions to the PS state. Shown are photon pair detection rates as a function of delay time for one or the other contribution indicating a crossover in source brightness at $\tau=15 \mathrm{~ns}$, which corresponds to balanced $|H H\rangle,|V V\rangle$ amplitudes and maximum concurrence. (d) Comparison between the theoretical concurrence (surface) and the experimental observations (filled squares) for a coincidence time window of $26 \mathrm{~ns}$ centered on $\tau=0$. The contour plot and the empty squares on the bottom plane are the projection of the theoretical surface and of the experimental data on the space of SV and CS photon fluxes, $\Phi_{S}$ and $\Phi_{C}$, respectively. The upper empty squares lie on the surface and represent the expected concurrence for the measured density matrices. Gray bars indicate $\pm 1 \sigma$ statistical errors calculated by bootstrapping. For all cases, we obtain theoretical predictions by integrating the elements of the expected density matrix $\rho$ over the time window considered. 
in the phase stabilization on the time scale of the acquisition, would reduce the coherences and, thus, the entanglement.

Figure 1(d) shows measured concurrence at several values of $\Phi_{C}$ and $\Phi_{S}$ with $|\tau|<13$ ns and confirms other aspects of the predicted relationship between squeezing and particulate entanglement. First, in all cases, a statistically significant entanglement is observed. Second, the concurrence decreases away from the maximum entanglement area described in Eq. (3), either if the $\Phi_{C}$ and $\Phi_{S}$ components are imbalanced or if $\Phi_{S}$ increases beyond the squeezing bandwidth. It is perhaps surprising that more squeezing can give less entanglement. This is, however, required by entanglement monogamy: each photon is entangled with all of its coarrivees, but the total entanglement (concurrence) is limited. Hence, the entanglement with any given other photon must decrease.

We report the first particle-by-particle measurements on a macroscopic quantum state. We analyze the joint polarization state of photon pairs extracted randomly from a beam of polarization-squeezed light, an archetypal macroscopic quantum system analogous to squeezed states in spin-1/2 atoms. We confirm several predictions of recent spin-squeezing theory [18], including strong entanglement, with concurrence up to 0.7 , among all pairs of photons arriving within the squeezing coherence time, NooN-type entanglement, and concurrence that decreases with photon flux as required by entanglement monogamy. The technique, thus proven, can be applied to particle-by-particle studies of entanglement in interacting and/or computationally intractable bosonic systems, e.g., photon BECs [21], exciton polaritons [22], and Rydberg-blockade-bound photon gases [24].

We thank Raymond Y. Chiao and Jack Boyce for early discussions, Geoff Lundeen for early exploratory experiments, and David Paredes-Barato for useful discussions. This work was supported by the Spanish MINECO project MAGO (Ref. No. FIS2011-23520), by the European Research Council project AQUMET, and by Fundació Privada CELLEX. J.Z. was supported by the FI-DGR Ph.D. fellowship program of the Generalitat of Catalonia. Y. A. d. I. A. was supported by the Scholarship No. BES2009-017461, under the Spanish MINECO Project No. FIS2007-60179.

*morgan.mitchell@icfo.es

[1] I. M. Georgescu, S. Ashhab, and F. Nori, Rev. Mod. Phys. 86, 153 (2014).

[2] J. Bardeen, L. N. Cooper, and J. R. Schrieffer, Phys. Rev. 106, 162 (1957).

[3] P. W. Anderson, Science 235, 1196 (1987).

[4] A. Sørensen, L.-M. Duan, J. I. Cirac, and P. Zoller, Nature (London) 409, 63 (2001).

[5] A. S. Sørensen and K. Mølmer, Phys. Rev. Lett. 86, 4431 (2001).
[6] I. Bloch, Nature (London) 453, 1016 (2008).

[7] W. S. Bakr, J. I. Gillen, A. Peng, S. Folling, and M. Greiner, Nature (London) 462, 74 (2009).

[8] J. F. Sherson, C. Weitenberg, M. Endres, M. Cheneau, I. Bloch, and S. Kuhr, Nature (London) 467, 68 (2010).

[9] O. Gühne and G. Tóth, Phys. Rep. 474, 1 (2009).

[10] G. Vitagliano, P. Hyllus, I. L. Egusquiza, and G. Tóth, Phys. Rev. Lett. 107, 240502 (2011).

[11] C. Gross, T. Zibold, E. Nicklas, J. Estève, and M. K. Oberthaler, Nature (London) 464, 1165 (2010).

[12] N. Behbood, F. Martin Ciurana, G. Colangelo, M. Napolitano, G. Tóth, R. J. Sewell, and M. W. Mitchell, Phys. Rev. Lett. 113, 093601 (2014).

[13] T. Monz, P. Schindler, J. T. Barreiro, M. Chwalla, D. Nigg, W. A. Coish, M. Harlander, W. Hänsel, M. Hennrich, and R. Blatt, Phys. Rev. Lett. 106, 130506 (2011).

[14] X.-C. Yao, T.-X. Wang, P. Xu, H. Lu, G.-S. Pan, X.-H. Bao, C.-Z. Peng, C.-Y. Lu, Y.-A. Chen, and J.-W. Pan, Nat. Photonics 6, 225 (2012).

[15] K. D. Nelson, X. Li, and D. S. Weiss, Nat. Phys. 3, 556 (2007).

[16] R. Bücker, A. Perrin, S. Manz, T. Betz, C. Koller, T. Plisson, J. Rottmann, T. Schumm, and J. Schmiedmayer, New J. Phys. 11, 103039 (2009).

[17] D. F. V. James, P. G. Kwiat, W. J. Munro, and A. G. White, Phys. Rev. A 64, 052312 (2001).

[18] F. A. Beduini and M. W. Mitchell, Phys. Rev. Lett. 111, 143601 (2013).

[19] N. Korolkova, in Quantum Information with Continuous Variables of Atoms and Light, edited by N. J. Cerf, G. Leuchs, and E.S. Polzik (Imperial College Press, London, 2007), Chap. 10, pp. 181-196.

[20] R. B. A. Adamson, L. K. Shalm, M. W. Mitchell, and A. M. Steinberg, Phys. Rev. Lett. 98, 043601 (2007).

[21] J. Klaers, J. Schmitt, F. Vewinger, and M. Weitz, Nature (London) 468, 545 (2010).

[22] M. Abbarchi, A. Amo, V. G. Sala, D. D. Solnyshkov, H. Flayac, L. Ferrier, I. Sagnes, E. Galopin, A. Lemaitre, G. Malpuech, and J. Bloch, Nat. Phys. 9, 275 (2013).

[23] E. E. Mikhailov and I. Novikova, Opt. Lett. 33, 1213 (2008).

[24] O. Firstenberg, T. Peyronel, Q.-Y. Liang, A. V. Gorshkov, M. D. Lukin, and V. Vuletic, Nature (London) 502, 71 (2013).

[25] J. Schmitt, T. Damm, D. Dung, F. Vewinger, J. Klaers, and M. Weitz, Phys. Rev. Lett. 112, 030401 (2014).

[26] J. Klaers, J. Schmitt, T. Damm, D. Dung, F. Vewinger, and M. Weitz, Proc. SPIE Int. Soc. Opt. Eng. 8600, 86000L (2013).

[27] A. Predojević, Z. Zhai, J. M. Caballero, and M. W. Mitchell, Phys. Rev. A 78, 063820 (2008).

[28] See Supplemental Material at http://link.aps.org/ supplemental/10.1103/PhysRevLett.114.120402 for a complete description of the experimental setup and of the data analysis.

[29] J. A. Zielińska, F. A. Beduini, N. Godbout, and M. W. Mitchell, Opt. Lett. 37, 524 (2012).

[30] J. A. Zielińska, F. A. Beduini, V. G. Lucivero, and M. W. Mitchell, Opt. Express 22, 25307 (2014).

[31] A NooN state is a two-mode entangled state with the form $\left|N_{H} 0_{V}\right\rangle+e^{i N \phi}\left|0_{H} N_{V}\right\rangle$. 